\title{
Neutral and Charged Excitons Localized in the InAs/GaAs Wetting Layer
}

\author{
A. Babiński ${ }^{a, *}$, M. Czyż ${ }^{a}$, J. Borysiuk ${ }^{b}, \mathrm{~S} \mathrm{KReT}^{c}$, \\ A. GolniK ${ }^{a}$, S. RAYMOnd ${ }^{d}$, J. LAPOINTE ${ }^{d}$ AND Z.R. \\ WASILEWSKI $^{d}$ \\ ${ }^{a}$ Institute of Experimental Physics, University of Warsaw \\ Hoża 69, 00-681 Warszawa, Poland \\ ${ }^{b}$ Institute of Electronic Materials Technology \\ Wólczyńska 133, 01-919 Warszawa, Poland \\ ${ }^{c}$ Institute of Physics, Polish Academy of Sciences \\ al. Lotników 32/46, 02-668 Warszawa, Poland \\ ${ }^{d}$ Institute for Microstructural Sciences, NRC \\ Ottawa, Ontario, K1A 0R6, Canada
}

It has recently been shown that potential fluctuations in a wetting layer, which accompanies InAs/GaAs quantum dots can localize excitons. Neutral excitons and biexcitons and charged excitons were identified. In this communication we report on studies of properties of the excitons over wide temperature range $(T<70 \mathrm{~K})$. The micro-photoluminescence measurements enable investigation of excitons localized in a single potential fluctuation. Temperature-induced broadening of the neutral exciton as well as a quenching of the charged exciton at temperatures higher than $50 \mathrm{~K}$ are observed and discussed.

PACS numbers: 73.21.La, 78.55.Cr, 78.67.Hc

\section{Introduction}

The most intensely investigated semiconductor structures, which provide a three-dimensional localization of excitons are self-assembled quantum dots (QDs) [1]. Potential fluctuations, which confine excitons are also present in disordered thin quantum wells $[2,3]$. We have recently shown that excitons can also be confined in a wetting layer (WL), which accompanies InAs/GaAs self-assembled QDs [4]. In this communication we present results of more detailed studies of properties of the confined excitons.

*corresponding author; e-mail: babinski@fuw.edu.pl 


\section{Experimental procedure}

The sample investigated in this work was grown by molecular beam epitaxy [5]. It contains a single layer of InAs QDs grown at $524^{\circ} \mathrm{C}$ on $n^{+} \mathrm{GaAs}$ substrate and $800 \mathrm{~nm}$ GaAs buffer layer and capped with $100 \mathrm{~nm}$ GaAs top layer. In-flush has been applied to the QDs at $5 \mathrm{~nm}$. A set of mesa structures were prepared on the sample in order to reproducibly locate a dot, addressed optically. The He-Cd laser $(\lambda=442 \mathrm{~nm})$ was used for photoluminescence (PL) excitation. The sample was placed in a continuous-flow cryostat and PL measurements were performed in temperature range $T=4.2-70 \mathrm{~K}$. The excitation light was delivered and the PL was collected via a microscope objective (spot size $\approx 1 \mu \mathrm{m}$ ), dispersed using a $0.5 \mathrm{~m}$ monochromator and detected by a CCD camera.

\section{Results and discussion}

The PL spectrum from the investigated structure consists of the emission from the GaAs barrier and self-assembled QD [6], as well as the emission related to the WL. The typical PL spectrum in the energy range of the expected WL-emission measured on a submicron-size mesa $(600 \mathrm{~nm} \times 600 \mathrm{~nm})$ consists of several sharp lines, observed in the energy range $1.42-1.44 \mathrm{eV}$ (see Fig. 1). The features are

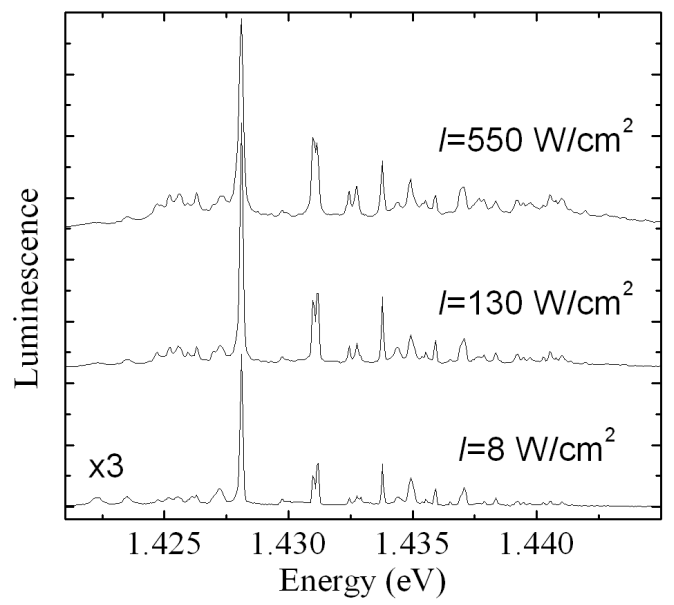

Fig. 1. Micro-PL spectra in the WL luminescence energy range $(T=6 \mathrm{~K})$ from a $600 \mathrm{~nm} \times 600 \mathrm{~nm}$ mesa with self-assembled InAs/GaAs QDs.

related to recombination of electrons and heavy holes localized in the InAs/GaAs WL [7]. The discrete structure of the emission shows that the WL is strongly disordered. The disorder leads to potential fluctuations, which localize excitons, as previously observed in thin GaAs/AlGaAs [8] or CdTe/CdMgTe [9] quantum wells. The spectrum measured on a larger mesa $(10 \mu \mathrm{m} \times 10 \mu \mathrm{m})$ evolves into the quasi-continuum of a broad peak. Moreover, at some spots on investigated 


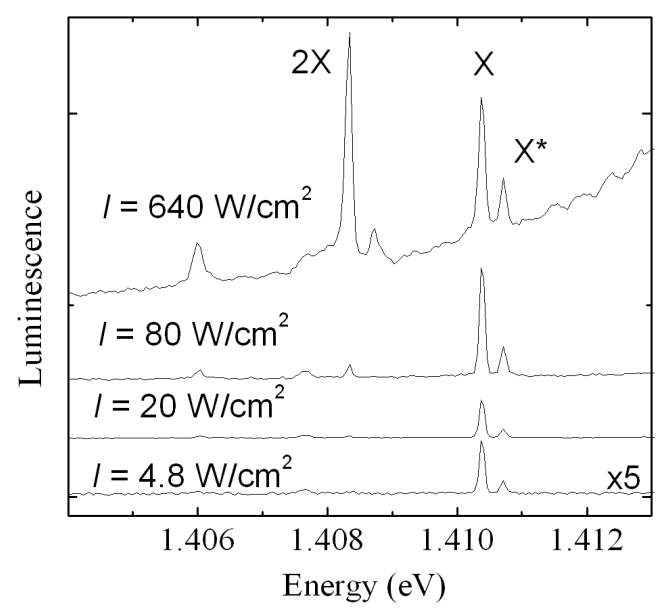

Fig. 2. Micro-PL spectra $(T=6 \mathrm{~K})$ in the WL luminescence energy range from a $10 \mu \mathrm{m} \times 10 \mu \mathrm{m}$ mesa with self-assembled InAs/GaAs QDs.

mesas, several well-resolved lines emerge in the spectrum at lower (up to $10 \mathrm{meV}$ ) energies. Emission lines from the low-energy tail of the spectrum must be due to recombination of excitons in the deepest potential fluctuations. Typical spectrum related to excitons confined in those fluctuations is shown in Fig. 2.

At low excitation power density the spectrum is dominated by two emission lines: $X$ and $X^{*}$. Both lines gain in intensity with increasing power and simultaneously another emission line $(2 X)$ emerges at lower energy (separated from $X$ by $2.0 \pm 0.1 \mathrm{meV}$ ). The $2 X$ emission line dominates the spectrum excited with the highest power density. The exciton occupancy for the $X, X^{*}$, and $2 X$ emission lines has been established by investigation of their intensity variation as a function of excitation density (see Fig. 3). Intensities of the $X$ and $X^{*}$ lines increase sublinearly before simultaneously reaching a maximum and decreasing for higher excitation density, which suggests that both lines arise from a single exciton occupancy of the potential fluctuation. The $2 X$ line intensity increases superlinearly, which confirms its attribution to the biexciton recombination.

The charge state of the excitons has been identified by performing polarization-sensitive PL measurements [4]. The $X$ and $2 X$ emission lines are split into two components, which are linearly polarized in perpendicular directions. The splitting must result from anisotropic exchange electron-hole interaction [2], which allows us to attribute the emission lines to a neutral exciton and biexciton recombination in a QD. The optical asymmetry has not been observed in the case of the $X^{*}$ emission line. This suggests its attribution to a trion (charged exciton). Observation of both the neutral and charged excitons is usually reported in spectroscopic studies of QDs excited non-resonantly [10]. The charge state of the trion cannot be established. Inspection of tens of spectra from different mesas revealed that the binding energy of trions can be either negative or positive. 


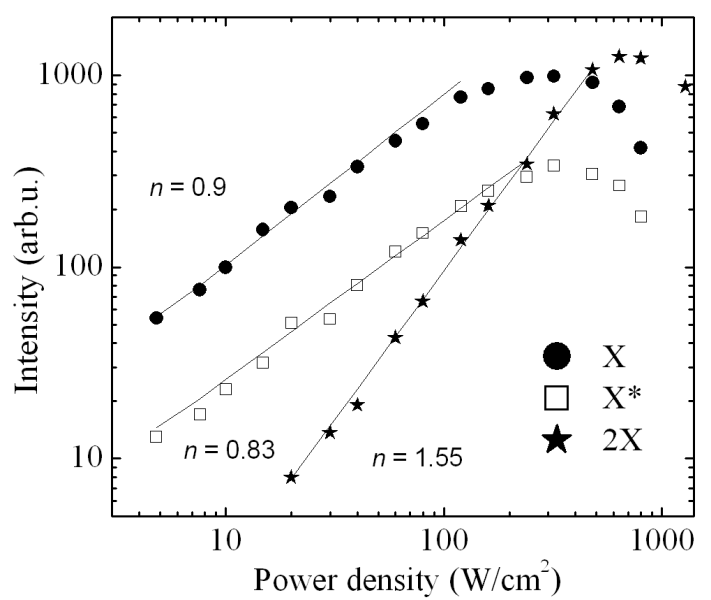

Fig. 3. Excitation-power dependence of the exciton $(X)$, biexciton $(2 X)$, and charged exciton $\left(X^{*}\right)$ intensity at $T=6 \mathrm{~K}$.

The attribution of $X$ and $X^{*}$ to neutral and charged excitons, respectively, can be further confirmed by PL investigations at elevated temperatures (see Fig. 4). It has been found that the $X^{*}$ emission line disappears from the spectrum at temperature $\approx 50 \mathrm{~K}$, while the $X$ emission line can be observed at temperatures up to $70 \mathrm{~K}$. The fast quenching of the $X^{*}$ line with respect to the $X$, observed at $>50 \mathrm{~K}$ is characteristic of a charged exciton and arises from the enhanced probability of an excess carrier escape from the ground state to higher energy states [11-13]. The temperature-dependent PL measurements also reveal the broadening of the emission lines as a function of temperature. The FWHM of the $X$ emission line at lowest temperatures is equal to $80 \mu \mathrm{eV}$ and it does not change significantly up to $T=20 \mathrm{~K}$ (see inset to Fig. 4). At higher temperatures the FWHM increases significantly. The broadening of the excitonic line at temperatures higher than $20 \mathrm{~K}$ correlates with a decrease in its intensity, which indicates that thermal activation of carriers to higher energy states is responsible for the broadening. The temperature dependence of the $X$ emission line-broadening can be reasonably well reproduced with a formula: $\Gamma(T)=\Gamma_{0}+A \exp \left(-E_{\mathrm{A}} / k T\right)[11]$ with $\Gamma_{0}=0.08 \mathrm{meV}$, and the activation energy $E_{\mathrm{A}}=12 \pm 2 \mathrm{meV}$ (see inset to Fig. 4). The activation energy most likely corresponds to a phonon-induced activation of a carrier from the ground state to the excited state.

In order to investigate the source of the fluctuations, responsible for the exciton confinement, the HRTEM analysis of the structure has been performed. It has been found that the WL is broadened. Moreover, the asymmetric shape of the In-composition profile has been observed, which is due to segregation of indium during the growth. The profile has been characterized using phenomenological $\mathrm{Mu}$ raki model of indium segregation [14]. As a result the nominal WL width 2.9 ML, and segregation coefficient $R=0.716$ have been obtained [4]. The indium compo- 


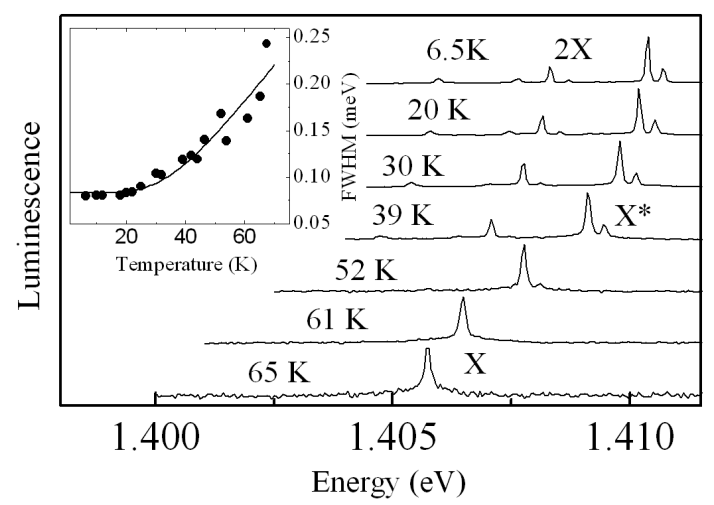

Fig. 4. Temperature dependence of the micro-PL spectra normalized for fixed excitation intensity $\left(103 \mathrm{~W} / \mathrm{cm}^{2}\right)$. In the inset: the temperature dependence of the PL line width of the neutral exciton $(X)$ and a fit of the form $\Gamma(T)=\Gamma_{0}+A \exp \left(-E_{\mathrm{A}} / k T\right)$, with $\Gamma_{0}=0.08 \mathrm{meV}$, and the activation energy $E_{\mathrm{A}}=12 \pm 2 \mathrm{meV}$.

sition has also been found to fluctuate in the WL plane, which corresponds to the disordered structure of the WL [15]. These fluctuations affect the band structure by composition and strain-effects and give rise to three-dimensional localization of excitons in the WL.

\section{Conclusions}

In conclusion, we have shown that the WL in the structure with InAs/GaAs self-assembled QDs is strongly disordered. Potential fluctuations resulting from the disorder are proposed to confine single excitons. Neutral and charged excitons as well as neutral biexcitons were identified and their temperature dependence was investigated. It was found that the exciton FWHM does not change significantly up to $T=20 \mathrm{~K}$. The activation-like broadening of the excitonic emission line at higher temperatures was observed. It was proposed that In composition fluctuations observed by means of transmission electron microscopy are responsible for potential fluctuations, which confine investigated excitons.

\section{Acknowledgments}

The work was supported in part by the MTKD-CT-2005-029671 project from the EU. The access to the JEOL JEM 3010 electron microscope at the Faculty of Materials Science and Engineering of Warsaw University of Technology is kindly acknowledged.

\section{References}

[1] For review see D. Bimberg, M. Grundmann, N.N. Ledentsov, Quantum Dot Heterostructures, Wiley, New York 1999. 
[2] D. Gammon, E.S. Snow, B.V. Shanabrook, D.S. Katzer, D. Park, Phys. Rev. Lett. 76, 3005 (1996).

[3] M. Hugues, M. Teisseire, J.-M. Chauveau, B. Vinter, B. Damilano, J.-Y. Duboz, J. Massie, Phys. Rev. B 76, 075335 (2007).

[4] A. Babinski, M. Czyż, J. Borysiuk, S. Kret, A. Golnik, S. Raymond, Z.R. Wasilewski, Appl. Phys. Lett. 92, 171104 (2008).

[5] Z.R. Wasilewski, S. Fafard, J.P. McCaffrey, J. Cryst. Growth 201/202, 1131 (1999).

[6] For more details on the QDs emission see A. Babinski, M. Potemski, S. Raymond, J. Lapointe, Z. Wasilewski, Phys. Rev. B 74, 155301 (2006).

[7] S. Sanguinetti, M. Henini, M. Grassi Alessi, M. Caprozzi, P. Frigeri, S. Franchi, Phys. Rev. 60, 8276 (1999).

[8] M. Erdman, C. Ropers, M. Wenderoth, R.G. Ulbricht, S. Malzer, G.H. Döhler, Phys. Rev. B 74, 125412 (2006).

[9] L. Besombes, K. Kheng, D. Martrou, Phys. Rev Lett. 85, 425 (2000).

[10] J.J. Finley, A.D. Ashmore, A. Lemaître, D.J. Mowbray, M.S. Skolnick, I.E. Itskevich, P.A. Maksym, M. Hopkinson, T.F. Krauss, Phys. Rev. B 63, 073307 (2001).

[11] R. Oulton, A.I. Tartakovskii, A. Ebbens, J. Cahill, J.J .Finley, D.J. Mowbray, M.S. Skolnick, M. Hopkinson, Phys. Rev. B 69, 155323 (2004).

[12] L. Besombes, K. Kheng, L. Marsal, H. Mariette, Phys. Rev. B 63, 155307 (2001).

[13] M. Bayer, A. Forchel, Phys. Rev. B 65, 041308(R) (2002).

[14] K. Muraki, S. Fukatsu, Y. Shiraki, Appl. Phys. Lett. 61, 557 (1992).

[15] V. Turck, F. Heinrichsdorff, M. Veit, R. Heitz, M. Grundmann, A. Krost, D. Bimberg, Appl. Surf. Sci. 123/124, 352 (1998). 